\title{
IMPLEMENTACIÓN DE FICHAS DIDÁCTICAS PARA EL TRABAJO INTERDISCIPLINARIO EN LA ESCUELA PRIMARIA EN CONTEXTO DE PANDEMIA
}

\author{
IMPLEMENTAÇÃO DE CARTOES DE ENSINO PARA O TRABALHO \\ INTERDISCIPLINAR NA ESCOLA PRIMÁRIA NO CONTEXTO DA \\ PANDEMIA
}

\author{
IMPLEMENTATION OF DIDACTIC WORKSHEETS FOR \\ INTERDISCIPLINARY WORK IN THE PRIMARY SCHOOL IN THE \\ CONTEXT OF PANDEMIC
}

DOI: $10.22481 /$ rbba.v10i02.9476

Magali Freyre

Universidad Nacional del Litoral, Santa Fe, Santa Fe, Argentina ORCID: https://orcid.org/ 0000-0003-4195-2940 Dirección electrónica: magali.freyre@gmail.com

María Silvina Reyes Universidad Nacional del Litoral, Santa Fe, Santa Fe, Argentina ORCID: https://orcid.org/0000-0002-1035-2769 Dirección electrónica: mariasilvinareyes@ hotmail.com

\begin{abstract}
RESUMEN
A partir del aislamiento social obligatorio dispuesto como medida sanitaria ante la pandemia de COVID-19, la actividad educativa presencial fue suspendida en todos sus niveles. En este contexto, se debió recurrir a la educación virtual, constituyendo nuevos modos de enseñar y aprender con el objetivo de garantizar la continuidad de los procesos educativos. Desde el Programa de Extensión Educación y Sociedad. Hacia una Mayor Inclusión Educativa, perteneciente a la Secretaría de Extensión Social y Cultural
\end{abstract}


de la Universidad Nacional del Litoral, Santa Fe Argentina se desarrolla una propuesta destinada a contribuir a la enseñanza remota en la educación primaria, entendiendo a la educación científica como un imperativo estratégico. Se presenta el análisis de los procesos de: diseño de fichas didácticas con propuestas interdisciplinarias para trabajar contenidos de Matemática y Ciencias Naturales a partir de actividades experimentales simples, difusión de las fichas en redes sociales, charla virtual y página web; y evaluación de la implementación de las fichas por parte de docentes, a través de los datos recogidos en un cuestionario. La evaluación referida a la aplicación de las fichas por parte de docentes fue positiva, de acuerdo a las evidencias que proveen las opiniones recabadas en el cuestionario.

Palabras clave: Fichas didácticas. Interdisciplina. Escuela primaria. Pandemia.

\section{RESUMO}

No momento do isolamento social obrigatório fornecido como medida de saúde em face da pandemia COVID-19, a atividade educacional cara a cara foi suspensa em todos os níveis. Nesse contexto, foi necessário apelar à educação virtual, constituindo-se em novas formas de ensino e aprendizagem para garantir a continuidade dos processos educativos. Desde o Programa de Extensão Educação e Sociedade. Rumo a uma Maior Inclusão Educacional, pertencente à Secretaria de Extensão Social e Cultural da Universidad Nacional do Litoral, Santa Fé - Argentina, desenvolve-se uma proposta para contribuir com o ensino a distância na educação básica, entendendo a educação científica como um imperativo estratégico.

A análise dos processos de: elaboração de arquivos didáticos com propostas interdisciplinares para trabalhar conteúdos de Matemática e Ciências Naturais é apresentada a partir de atividades experimentais simples, divulgação dos cartoes em redes sociais, chat virtual e página web; e avaliação da aplicação dos arquivos pelos professores, por meio dos dados coletados em um questionário. A avaliação quanto à aplicação dos cartões pelos professores foi positiva, de acordo com as evidências fornecidas pelas opiniões coletadas no questionário.

Palavras-chave: Cartões de ensino. Interdisciplinar. Escola primária. Pandemia. 


\begin{abstract}
Based on the mandatory social isolation provided as a health measure in the face of the COVID-19 pandemic, face-to-face educational activity was suspended at all levels. In this context, virtual education had to be developed, constituting new ways of teaching and learning to guarantee the continuity of educational processes. From the Extension Program called Educación y Sociedad. Hacia una mayor inclusion Educativa, belonging to the Secretaría de Extensión Social y Cultural of the Universidad Nacional del Litoral, Santa Fe - Argentina, a proposal is developed to contribute to remote teaching in primary education, understanding scientific education as an imperative strategy. The analysis of the processes of design of didactic worksheets with interdisciplinary proposals to work on Mathematics and Natural Sciences contents from simple experimental activities, dissemination of the worksheets on social networks, virtual meeting and web page and evaluation of the implementation of the worksheets by teachers, through the data collected in a questionnaire, is presented. The evaluation referring to the application of the worksheets by teachers was positive, according to the evidence provided by the opinions collected in the questionnaire.
\end{abstract}

Keywords: Didactic worksheets. Interdisciplinary. Primary school. Pandemic.

\title{
INTRODUCCIÓN
}

La pandemia de COVID- 19 y el aislamiento social obligatorio dispuesto en gran parte de los países como medida sanitaria para evitar el aumento exponencial de contagios, obligó a la población a modificar sus modos de vida y resultó imprescindible buscar caminos alternativos para adaptar ciertas actividades cotidianas a una nueva realidad social. En este marco, la actividad educativa presencial fue suspendida en todos sus niveles y se debió recurrir a la educación virtual, con el objetivo de garantizar la continuidad de los procesos educativos, con la certeza de que el regreso a las aulas y laboratorios se extendería en el tiempo (CAYOROJAS y AGRAMONTE-ROSELL, 2020). Frente a este escenario, nuevos modos de enseñar y aprender se hicieron presentes. Como afirma Maggio (2021), dado que no se puede educar en una pandemia haciendo de cuenta que no existe, fue fundamental diseñar enfoques nuevos considerando lo prioritario desde una perspectiva curricular; lo que exigió una nueva Revista RBBA $\quad$ Revista Binacional Brasil Argentina Vol. 10, num. 2, dez/2021, p. 173-184 
interpretación sobre lo que ya estaba acordado. En este sentido, desde el Programa de Extensión "Educación y Sociedad. Hacia una Mayor Inclusión Educativa", perteneciente a la Secretaría de Extensión Social y Cultural (SESyC) de la Universidad Nacional del Litoral, Santa Fe Argentina, se pensaron una serie de acciones destinadas a docentes de nivel primario con la premisa de contribuir a la enseñanza remota de las Ciencias Naturales y la Matemática, entendiendo que la educación científica es un imperativo estratégico. La participación plena en la sociedad actual requiere, que la ciudadanía tenga una formación que le permita comprender y actuar sobre un mundo cada vez más complejo, cambiante y profundamente impregnado por la ciencia y la tecnología (FURMAN, 2016). En esta línea, existe también un acuerdo cada vez mayor acerca del papel que desempeña la educación científica y tecnológica en la promoción de capacidades relacionadas con la innovación, el aprendizaje continuo y el pensamiento crítico desde edades tempranas (HARLEN, 2008).

Una de estas acciones fue diseñar fichas didácticas que incluyeran actividades experimentales simples (AES). En primer lugar, se hará referencia a las características que presentan las actividades experimentales, teniendo en cuenta que son acciones o situaciones donde el resultado, si bien es desconocido (y hasta sorprendente para el estudiantado) está predeterminado por una teoría consensuada científicamente, planificado didácticamente y cuyo objetivo primordial es que las y los estudiantes aprendan algún contenido (seleccionado intencionalmente) de química, de biología o de física como así también alguna técnica, destreza o actitud (REVERDITO y LORENZO, 2007). De este modo, las AES comparten las características de las actividades experimentales, pero la diferencia es que pueden implementarse tanto en aulas convencionales como en diferentes espacios. En otras palabras, las actividades experimentales simples, no necesitan de un laboratorio para su realización. En este sentido, es posible que los estudiantes aborden las AES propuestas sin que esto conlleve dificultades para su realización en la casa. Esto se desarrolla teniendo en cuenta que el apoyo o ayuda que brindan las familias a los estudiantes no se da de manera homogénea, sino que dependen de ciertas posibilidades con las que cuentan (MAGGIO, 2021). Las actividades experimentales simples que se presentan en algunas fichas articulan desde una perspectiva interdisciplinaria contenidos de Matemática y Ciencias Naturales. La mirada del enfoque interdisciplinario se convierte en una eficaz estrategia, capaz de agrupar una amplia variedad de prácticas educativas que se desarrollan en las aulas o en otros escenarios educativos, teniendo en cuenta que cuanto mayor bagaje de saberes propios de otras disciplinas se puedan poner en 


\section{IMPLEMENTACIÓN DE FICHAS DIDÁCTICAS PARA EL TRABAJO \\ INTERDISCIPLINARIO EN LA ESCUELA PRIMARIA EN CONTEXTO DE}

PANDEMIA

interacción, más amplitud se alcanzará en el análisis de una determinada problemática (TORRES SANTOMÉ, 2006).

En consonancia a lo expuesto anteriormente, desde el Ministerio de Educación de la Provincia de Santa Fe, en el año 2016, se presentaron los Núcleos Interdisciplinarios de Contenidos (NIC). Este documento regulatorio de desarrollo curricular para la educación primaria y secundaria sugiere que las propuestas de enseñanza se trabajen superando la fragmentación de saberes, desde una concepción integrada de los mismos.

Uno de los temas propuestos para ser trabajado desde esta perspectiva en los NIC es La Alimentación, como actividad propia de todo ser vivo y específica del ser humano. Se produce cultura a través de la alimentación, teniendo en cuenta las maneras de obtener alimento y las acciones que se desarrollan en relación con el mismo. Por esta razón, resultan de interés para trabajar con los estudiantes de escuela primaria y secundaria todas las actividades humanas que se llevan a cabo en torno a la alimentación. "La alimentación es importante para la supervivencia, pero también para el encuentro con otro, para el cuidado de la salud, para el desarrollo de la vida misma" (p. 99). Algunos de los objetivos que se enuncian en relación a esta problemática son: indagar cómo están formados los alimentos y promover la matematización, las capacidades de comunicación y de pensamiento lógico matemático de los estudiantes a partir de materiales que permitan ejercitar y desarrollar estas competencias.

Teniendo en cuenta lo anterior, el presente trabajo plantea como propósito realizar un análisis de los procesos de: diseño de fichas didácticas con propuestas interdisciplinarias, difusión de las fichas en redes sociales, charla virtual y página web; y evaluación de la implementación de las fichas por parte de las y los docentes.

\section{Desarrollo de la experiencia}

\section{Diseño de materiales: Fichas El laboratorio en casa}

El Laboratorio en casa consiste en una herramienta didáctica para la enseñanza de las Ciencias Naturales, generada en el contexto de la pandemia de COVID-2019, tal como se muestra en la Figura 1. Uno de los propósitos de este instrumento, fue acercar a los y las docentes propuestas de actividades experimentales simples para trabajar con los alumnos y alumnas desde la casa. En cada una de las fichas se detallan los contenidos curriculares a

Revista RBBA $\mid$ Revista Binacional Brasil Argentina Vol. 10, num. 2, dez/2021, p. 173-184 


\section{IMPLEMENTACIÓN DE FICHAS DIDÁCTICAS PARA EL TRABAJO \\ INTERDISCIPLINARIO EN LA ESCUELA PRIMARIA EN CONTEXTO DE \\ PANDEMIA}

trabajar, los materiales necesarios, los procedimientos y la explicación de los resultados, como así también una serie de consignas para seguir trabajando luego de la finalización de la actividad experimental. Cabe destacar que a cada una de las fichas se le asignó un nombre metafórico, dando cuenta de un uso connotativo del lenguaje (Figura 2).

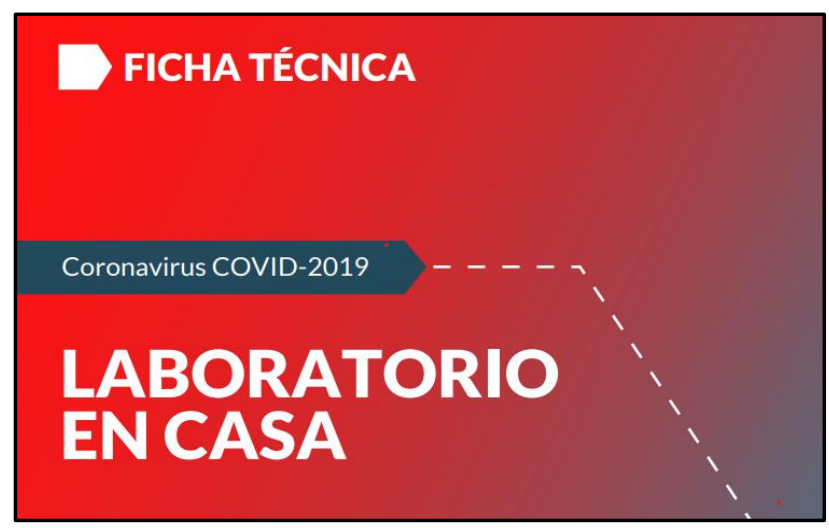

Figura 1. Fichas diseñadas en el marco de la pandemia COVID-2019

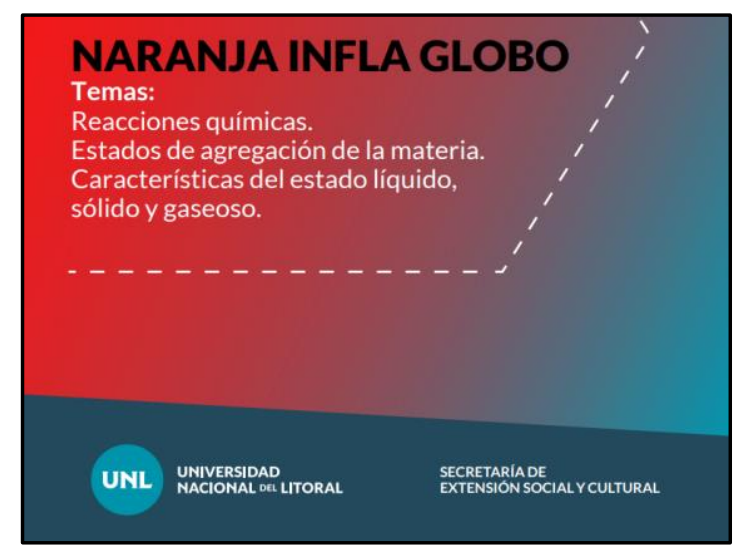

Figura 2. Ficha donde se aprecia el uso connotativo del lenguaje a través de un título metafórico

Dentro de la serie El laboratorio en casa, se encuentra una sección especial denominada Hacia el interior de los alimentos. En esta categoría las fichas desarrolladas abordan en forma específica contenidos de Matemática y Ciencias Naturales, trabajados de manera interdisciplinaria para la educación primaria. Es destacable que, en todos los casos, las actividades experimentales simples se realizan con materiales inocuos y de fácil accesibilidad. Las fichas que forman parte de este apartado "invitan" a conocer los alimentos desde su interior, a partir de la composición química de los mismos.

Revista RBBA $\quad$ Revista Binacional Brasil Argentina Vol. 10, num. 2, dez/2021, p. 173-184 


\section{IMPLEMENTACIÓN DE FICHAS DIDÁCTICAS PARA EL TRABAJO INTERDISCIPLINARIO EN LA ESCUELA PRIMARIA EN CONTEXTO DE \\ PANDEMIA}

Específicamente, una de las fichas se refiere a carbohidratos (Hacia el interior de los alimentos: Carbohidratos) y desarrolla además los siguientes contenidos:

Cambios químicos. Números decimales. Relaciones de proporcionalidad directa. La proporcionalidad como razón. La proporcionalidad como porcentaje (Figuras 3 y 4).

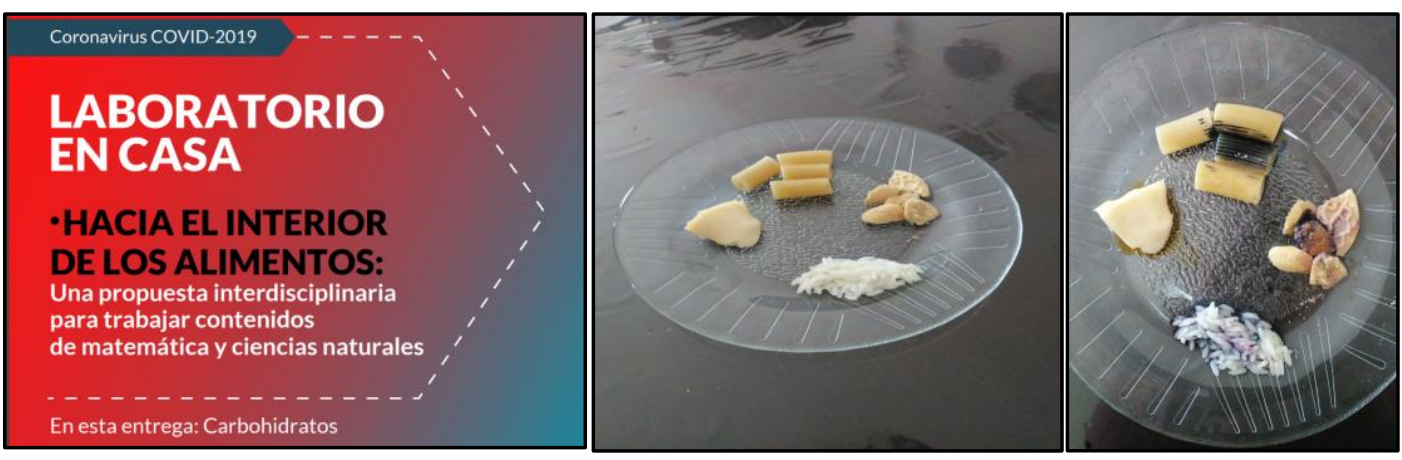

Figura 3. Determinación cualitativa de almidón en diferentes muestras alimentarias

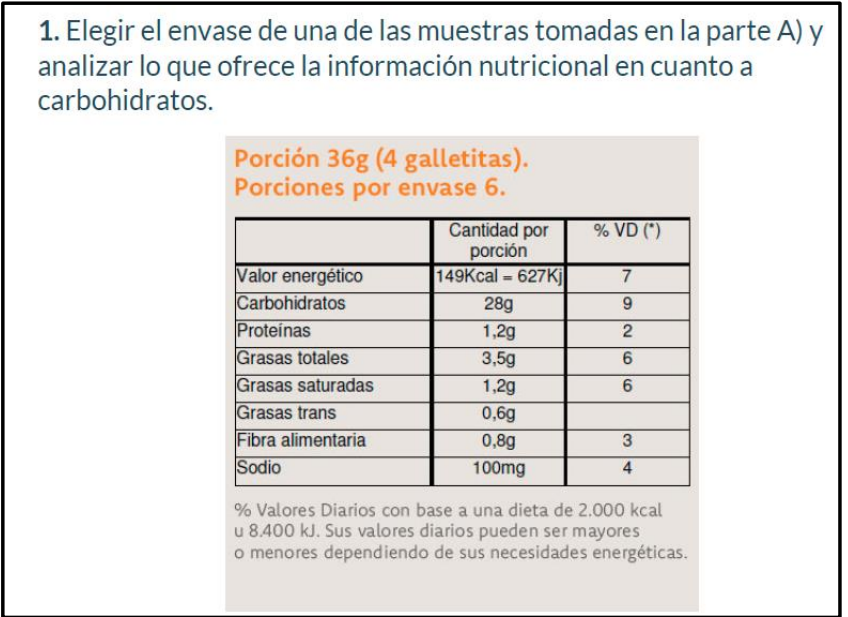

Para calcular el porcentaje se puede proceder de distintas maneras, una es calculando primero la razón que hay entre el total de carbohidratos y el total del producto.

2. En el ejemplo, hay $28 \mathrm{~g}$ de carbohidratos en un total de $36 \mathrm{~g}$ de producto. Entonces, la razón buscada es:

$$
\frac{28 g}{36 g}=0,8
$$

3. Para calcular el porcentaje se multiplica esa razón por 100. Lo que da como resultado que el producto tiene un porcentaje de carbohidratos del $80 \%$.

Figura 4. Razón y porcentaje en información nutricional.

Por otro lado, la ficha Hacia el interior de los alimentos: Lípidos, hace referencia a otro macronutriente como son los lípidos y desarrolla específicamente los siguientes contenidos: 


\section{Características de los lípidos. Características de los cuerpos geométricos. Cubrimiento del espacio (Figuras 5 y 6).}

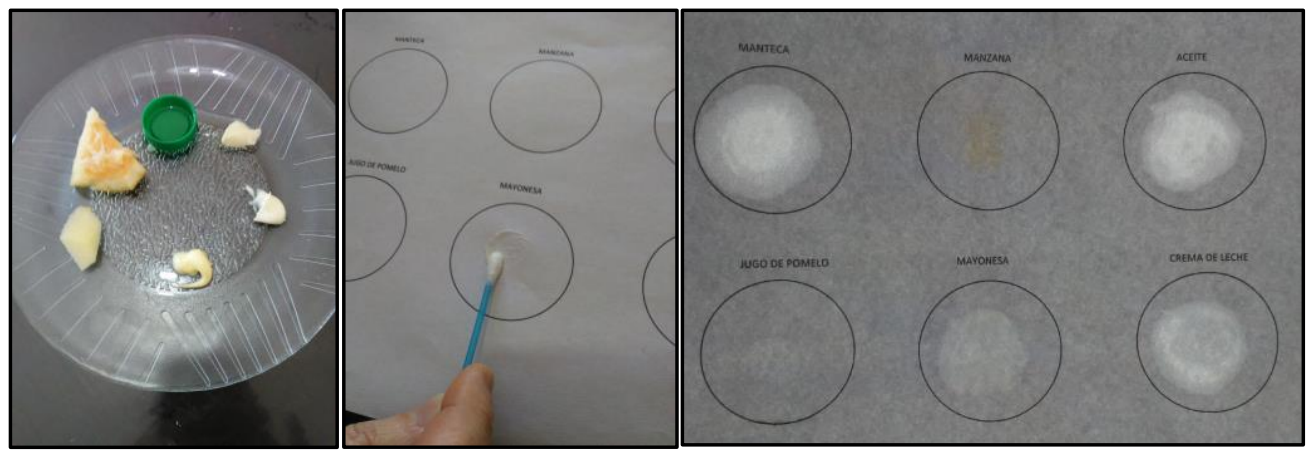

Figura 5. Determinación cualitativa de lípidos en diferentes muestras alimentarias

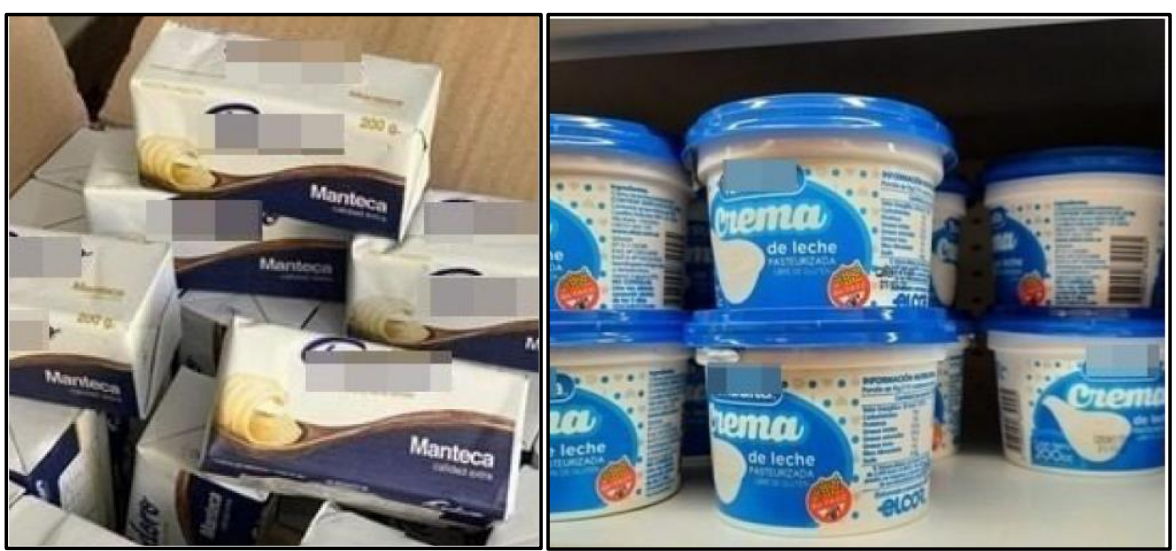

Figura 6. Cuerpos geométricos y cubrimiento del espacio

Difusión de las fichas

Las fichas fueron difundidas a través de las redes sociales Whatsapp y Facebook. Además, se encuentran disponibles en la página web de la Universidad Nacional del Litoral, desde la que pueden ser descargadas. Una de las fichas (Hacia el interior de los alimentos: Carbohidratos) fue presentada en forma sincrónica mediante una charla a partir de la plataforma Zoom, a través de la licencia adquirida por la SESyC, de la cual participaron más de un centenar de docentes de diferentes localidades de la provincia. Es destacable la posibilidad que brindaron los encuentros sincrónicos a través de plataformas de este tipo a las y los docentes, que pudieron estar conectados y reunirse especialmente para trabajar en equipo, cuestiones que en algunos casos no habían sido posible antes de la pandemia (MAGGIO, 2021). El gran número de parti- 
cipantes que asistió a la charla en la que se presentó la ficha, se debe en gran parte a su carácter virtual, ya que fue posible que se conecten desde distintas localidades, sin organización previa de viaje.

En la charla sincrónica se hizo referencia a la serie El laboratorio en casa aclarando a las y los participantes que todas las fichas quedaron disponibles en la página web de la Universidad Nacional del Litoral, aunque se profundizó especialmente sobre la ficha mencionada anteriormente. Como sostiene Maggio (2021, p. 99), las prácticas de enseñanza que adquieren carácter público expanden su relevancia y alcance. Así, "lo que ocurre en el aula, queda en el aula es solo otra frase más que ha perdido sentido".

\section{Evaluación de la implementación:}

a) Características de cuestionario enviado

Se invitó a las y los participantes de la charla a contestar una encuesta en fecha cercana a la finalización del ciclo lectivo 2020, cuyo objetivo era realizar un relevamiento sobre la utilización de las fichas y conocer su opinión en relación a éstas. El instrumento utilizado para la recolección de la información fue una encuesta anónima confeccionada en Google Forms. El formulario fue enviado a través de correo electrónico a las y los participantes de la charla, quienes de manera previa a contestar el mismo, manifestaron su consentimiento informado. Este instrumento consta de cinco preguntas de opción múltiple y una pregunta abierta.

Dentro de las preguntas de opción múltiple, algunas se refieren a ciertos datos personales del desempeño docente y otras a la implementación de las fichas. En la Tabla 1, se visualizan las primeras cinco preguntas contenidas en el formulario.

\begin{tabular}{|l|l|}
\hline Datos personales & $\begin{array}{l}\text { Pregunta 1: } \\
\text { ¿En qué nivel te desempeñas como docente? }\end{array}$ \\
\cline { 2 - 3 } & $\begin{array}{l}\text { Pregunta 2: } \\
\text { Gestión a la que corresponde el establecimiento educativo en el } \\
\text { que se desempeña. }\end{array}$ \\
\hline Implementación de las fichas & $\begin{array}{l}\text { Pregunta 3: } \\
\text { ¿Has utilizado las fichas? }\end{array}$ \\
\cline { 2 - 2 } & $\begin{array}{l}\text { Pregunta 4: } \\
\text { ¿Con cuál o cuáles de las fichas trabajaste? }\end{array}$ \\
\cline { 2 - 2 } & $\begin{array}{l}\text { Pregunta 5: } \\
\text { ¿Cómo calificarías los resultados obtenidos luego de haber } \\
\text { implementado las actividades propuestas en las fichas? }\end{array}$ \\
\hline
\end{tabular}

Tabla 1. Categorización y disposición de las preguntas en el cuestionario Revista RBBA $\quad$ Revista Binacional Brasil Argentina Vol. 10, num. 2, dez/2021, p. 173-184 


\section{IMPLEMENTACIÓN DE FICHAS DIDÁCTICAS PARA EL TRABAJO \\ INTERDISCIPLINARIO EN LA ESCUELA PRIMARIA EN CONTEXTO DE}

PANDEMIA

La pregunta seis, fue opcional y de respuesta abierta. Ofreció la posibilidad de escribir comentarios o sugerencias relacionadas a las preguntas anteriores.

b) Respuestas obtenidas del cuestionario

Con respecto al cuestionario enviado, once participantes de la charla lo respondieron. Vale destacar que no se pretende realizar generalizaciones a partir de las respuestas obtenidas, sino presentar una descripción de las mismas.

Para la primera pregunta se dieron como opciones los niveles primario, secundario y terciario, con la opción de responder a más de una. Se obtuvieron ocho respuestas de nivel primario, cuatro que refieren a nivel secundario y tres que mencionan nivel terciario. En la segunda pregunta debían optar por una, entre las opciones de gestión: pública o privada. Seis de los encuestados marcaron la opción pública y cinco la opción privada. El total de encuestados respondió que sí había utilizado las fichas.

En la cuarta pregunta, se obtuvieron ocho respuestas correspondientes a la ficha Hacia el interior de los alimentos: Carbohidratos, cuatro respuestas que marcan como opción Hacia el interior de los alimentos: Lípidos, seguidas de tres menciones de Naranja inflaglobo y dos relacionadas a la ficha Viraje de color (Figura 7).

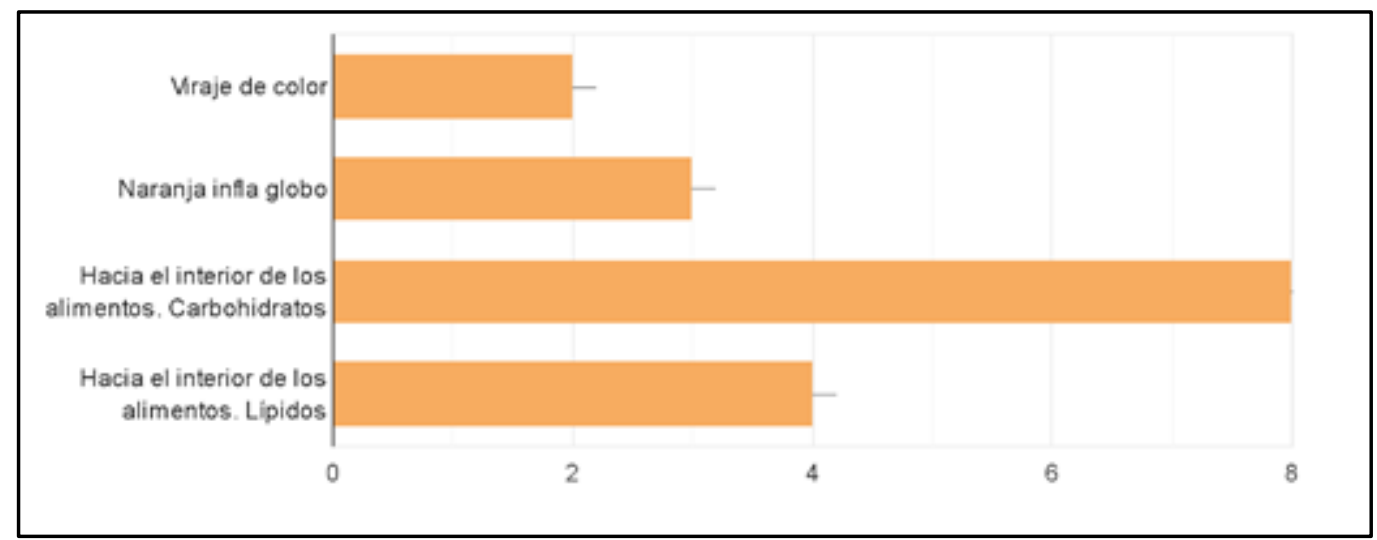

Figura 7. Respuestas obtenidas a partir de la pregunta 4

En la quinta pregunta se pidió que marquen solo una opción entre Muy satisfactorios, Satisfactorios y Regulares. Siete de los encuestados marcó la opción de Muy satisfactorios y el resto marcó la opción Satisfactorios. Por último, del total de participantes que realizaron la encuesta, siete escribieron comentarios en el espacio propuesto por la pregunta seis. Se pueden encontrar valoraciones positivas, destacando sencillez y significatividad del trabajo con experiencias, agradecimientos y en un caso la expresión de deseo acerca de continuar realizando instancias de formación.

Revista RBBA Revista Binacional Brasil Argentina Vol. 10, num. 2, dez/2021, p. 173-184 


\section{CONSIDERACIONES FINALES}

Teniendo en cuenta los objetivos planteados, se considera que los mismos han sido alcanzados. En este sentido, se diseñaron fichas didácticas con una perspectiva interdisciplinaria que pudieron ser utilizadas por estudiantes y docentes en períodos de aislamiento.

La difusión de las fichas se realizó a través de diferentes medios, no sólo a partir de dejar disponible la serie El Laboratorio en casa en una página web institucional, sino a través de redes sociales y Whatsapp. La experiencia del encuentro sincrónico de charla para la presentación de la ficha Hacia el interior de los alimentos: Carbohidratos representó otra posibilidad de dar a conocer el trabajo realizado. Resulta relevante que las AES correspondientes a dicha ficha fueron realizadas en el encuentro sincrónico, lo que facilitó la familiarización de los participantes de la charla con las actividades propuestas. La ficha presentada en la charla constituye la más nombrada dentro de las utilizadas por los participantes que respondieron el cuestionario.

Con respecto a la evaluación referida a la aplicación de las fichas por parte de las y los docentes puede decirse que fue positiva, de acuerdo a las evidencias que proveen las opiniones recabadas en el cuestionario.

Como afirma Maggio (2021), el tiempo que se atraviesa enfrenta no sólo a la problemática de la existencia de la humanidad sino a la necesidad de cambiar la realidad con premura. Las propuestas de enseñanza interdisciplinaria, por su parte, revalorizan el sentido de los aprendizajes más allá de la acumulación de fragmentos. Es que los procesos de aprendizaje genuino son impulsados a partir de las posibilidades de creación que brindan los problemas y las preguntas sin respuestas. De esta manera, las ansias de saber de docentes y estudiantes se suscitan a partir del deseo de comprender y transformar, cuestiones que pueden ser desarrolladas en pandemia.

¿Qué mejor oportunidad para querer aprender que la que ofrece una pandemia por más terrible que sea? Esto no remite sólo a las facultades de medicina o ciencias de la salud sino a todas las áreas del conocimiento y a todos los niveles del sistema educativo (MAGGIO, 2021, p.83)

El contexto de pandemia exige que las y los docentes modifiquen ciertas perspectivas y propongan prácticas que, aún atravesadas por los desafíos que presentan las características de

Revista RBBA Revista Binacional Brasil Argentina Vol. 10, num. 2, dez/2021, p. 173-184 
este tiempo, sean superadoras, renovadas, creativas e inclusivas. En este sentido, la experiencia desarrollada en el diseño, difusión y evaluación de El Laboratorio en casa, particularmente de la serie Hacia el interior de los alimentos, representa un ejemplo de propuesta que surge como fruto de reflexiones en torno a las prácticas de enseñanza que se pueden ofrecer ante una realidad que se impone con panorama incierto e inesperado.

\section{Agradecimientos:}

Las autoras agradecen al Programa de Extensión de la UNL, Educación y Sociedad. Hacia una mayor inclusión educativa, a su directora Mg. Ana María Mántica y a Sofía Soledad Espínola.

\section{REFERENCIAS}

CAYO-ROJAS, C. F. y AGRAMONTE-ROSELL, R. C. Desafíos de la educación virtual en odontología en tiempos de pandemia COVID-19. Revista Cubana de Estomatología, Cuba, v.57, n.3, p.1-3. 2020.

Disponible en: http://www.revestomatologia.sld.cu/index.php/est/article/view/3341 Acceso 25 may.2021.

FURMAN, M. Educar mentes curiosas: la formación del pensamiento científico y tecnológico en la infancia. XI Foro Latinoamericano de Educación. Santillana. Buenos Aires, 2016. 89p.

HARLEN, W. Teaching, learning and assessing science K-12. SAGE Publications, Londres. 2008. 263p.

MAGGIO, M. Educación en Pandemia. Paidós, Buenos Aires. 2020. 214p.

MINISTERIO DE EDUCACIÓN DE LA PROVINCIA DE SANTA FE. Núcleos interdisciplinarios de contenidos (NIC): Recursos para su enseñanza. Disponible en: https://campuseducativo.santafe.edu.ar/wp-content/uploads/PDF-Libro-NIC-II.pdf. Acceso 20 mayo 2021.

REVERDITO, A. M. y LORENZO, M. G. Actividades experimentales simples. Un punto de partida posible para la enseñanza de la química. Educación en la Química, Buenos Aires, v.13, n. 2, p.108-121. 2007.

TORRES SANTOME, J. Globalización e interdisciplinariedad: el curriculum integrado. Morata, Madrid. 2006. 280p. 